\title{
Reduction of glycine particle size by impinging jet crystallization
}

\author{
Tímea Tari, Zoltán Fekete, Piroska Szabó-Révész, Zoltán Aigner* \\ Department of Pharmaceutical Technology, University of Szeged, Eötvös u. 6, H-6720 Szeged, Hungary
}

\section{A R T I C L E I N F O}

\section{Article history:}

Received 25 June 2014

Received in revised form 7 November 2014

Accepted 8 November 2014

Available online 13 November 2014

\section{Keywords:}

Impinging jet crystallization

Particle size

Glycine

Crystal habit

Polymorphism

Residual solvent content

\begin{abstract}
A B S T R A C T
The parameters of crystallization processes determine the habit and particle size distribution of the products. A narrow particle size distribution and a small average particle size are crucial for the bioavailability of poorly water-soluble pharmacons. Thus, particle size reduction is often required during crystallization processes. Impinging jet crystallization is a method that results in a product with a reduced particle size due to the homogeneous and high degree of supersaturation at the impingement point.

In this work, the applicability of the impinging jet technique as a new approach in crystallization was investigated for the antisolvent crystallization of glycine. A factorial design was applied to choose the relevant crystallization factors. The results were analysed by means of a statistical program. The particle size distribution of the crystallized products was investigated with a laser diffraction particle size analyser. The roundness and morphology were determined with the use of a light microscopic image analysis system and a scanning electron microscope. Polymorphism was characterized by differential scanning calorimetry and powder X-ray diffraction. Headspace gas chromatography was utilized to determine the residual solvent content.

Impinging jet crystallization proved to reduce the particle size of glycine. The particle size distribution was appropriate, and the average particle size was an order of magnitude smaller $(d(0.5)=8-35 \mu \mathrm{m})$ than that achieved with conventional crystallization $(\mathrm{d}(0.5)=82-680 \mu \mathrm{m})$. The polymorphic forms of the products were influenced by the solvent ratio. The quantity of residual solvent in the crystallized products was in compliance with the requirements of the International Conference on Harmonization.
\end{abstract}

(c) 2014 Elsevier B.V. All rights reserved.

\section{Introduction}

Crystallization is an important pharmaceutical industrial process. The majority of active pharmaceutical ingredients (APIs) and excipients can be produced by crystallization. The crystallization process determines the chemical purity and physical properties of the product, including its habit, particle size and crystal structure. The average particle size, the particle size distribution, and the habit of particles play decisive roles in pharmaceutical formulation. These parameters may influence the bioavailability and the processability. Direct tablet compression requires sufficiently large and isodimensional particles, but a small average particle size with a narrow particle size distribution is preferred for poorly water-soluble APIs. The marketable materials will be those that can be directly applied in the formulation of pharmaceutical products (Hacherl et al., 2003; Woo et al., 2011; Liu et al., 2013).

\footnotetext{
* Corresponding author. Tel.: +36 62545 577; fax: +36 62545571 .

E-mail address: aigner@pharm.u-szeged.hu (Z. Aigner).
}

Crystallization methods that are commonly used in the pharmaceutical industry include cooling, antisolvent and precipitation processes. However, with these techniques the particle size can be reduced only within certain limits. New methods are therefore sought to decrease the particle size of APIs. One such may be sonocrystallization, which has been studied with various crystallization systems, but its advantages in various crystallization applications are disputed (McCausland et al., 2001; McCausland and Cains, 2003; Louhi-Kultanen et al., 2006). Other options involve the use of impinging jet crystallization and the application of multiple inlet vortex mixers (Liu et al., 2008; D'Addio and Prud'homme, 2011).

Midler et al. (1994) introduced and adapted the impinging jet technique in crystallization (Midler et al., 1994; Tung et al., 2009). The impinging jet mixer consists of two jet nozzles arranged diametrically opposite and facing each other. The impinging jet element can be used in a crystallization reactor or operated in nonsubmerged mode. A rich solution of the API and the antisolvent flow through the nozzles at a constant linear velocity, causing high supersaturation at the impingement point before the onset of nucleation. This process potentially results in rapid crystallization 
in the absence of concentration gradients and produces a monodisperse population of small crystals with a high surface area. Impinging jet crystallization is often used in combination with ultrasound to achieve a further reduction in particle size. The direct production of small uniform crystals with high surface area that meet the bioavailability and dissolution requirements can eliminate the need for milling, which can give rise to dust issues, yield losses, long production times, polymorphic transformation or amorphization (Woo et al., 2009; Bauer-Brandl, 1996a,b; am Ende and Brenek, 2004; Calvignac and Boutin, 2009; Hacherl et al., 2003; Dong et al., 2011).

Glycine exists in three polymorphic forms under ambient conditions. Forms $\alpha$ and $\beta$ are monoclinic $\left(\alpha, \mathrm{P} 2_{1} / \mathrm{n} ; \beta, \mathrm{P} 2_{1}\right)$, while $\gamma$ is trigonal $\left(\mathrm{P}_{1}\right)$. Other polymorphs have been observed at high pressure. In aqueous solution, form $\alpha$ is obtained by spontaneous nucleation (Rabesiaka et al., 2010; Goryainov et al., 2006; Lin et al., 1998). The less stable $\beta$ glycine has been found to transform rapidly into form $\alpha$ in air or water, but the crystals remain unchanged if kept in a dry environment. The $\gamma$ form of glycine is the stable form at room temperature and transforms to the $\alpha$ form when heated above $165^{\circ} \mathrm{C}$ (Boldyreva et al., 2003a,b,b; Ferrari et al., 2003; Srinivasan, 2008). The addition of ethanol to an aqueous glycine solution induces precipitation of the $\beta$ form (Weissbuch et al., 2005; Ferrari et al., 2003). The crystallization methods and conditions, the $\mathrm{pH}$ of the solution, and the presence of additives also influence the crystal morphology and the polymorphism (Dubbini et al., 2014).

Glycine is a widely used material for crystallization experiments (Srinivasan et al., 2011; Rabesiaka et al., 2010; LungSomarriba et al., 2004). It is fast-growing and its crystals are typically quite large, so it is a suitable model material for particle size reduction studies. In order to reduce the glycine particle size, Louhi-Kultanen et al. (2006) studied the effects of ultrasound during cooling crystallization on the polymorphism, crystal size distribution and heat transfer in batch cooling crystallization. Sonocrystallization proved to be a good tool for optimizing and controlling the nucleation and crystallization of glycine, and can be used as a size reduction method to produce a final product with uniform crystal morphology. The smallest average particle size achieved was about $100 \mu \mathrm{m}$. Aigner et al. (2012) examined the effects of several crystallization methods and their parameters (cooling, reverse antisolvent and antisolvent crystallization with ultrasound) on the average particle size, particle size distribution and roundness of glycine, and found that these methods are capable of reducing the average particle size only within a certain range. The particle size ranges $(\mathrm{d}(0.5))$ obtained were as follows: $268-680 \mu \mathrm{m}$ in cooling crystallization; $160-466 \mu \mathrm{m}$ in reverse antisolvent crystallization; and $82-232 \mu \mathrm{m}$ in antisolvent crystallization with ultrasound.

In the present work, the impinging jet antisolvent crystallization of glycine as model material were investigated by means of a factorial design for a further particle size decrease. The effects of a number of operating parameters, such as the linear velocity of feeding, the post-mixing time, the temperature difference and the solvent ratio, on the resulting particle size distribution and roundness were studied. A statistical program was used to evaluate the results. The particle size distribution was measured with a laser diffraction particle size analyser. Glycine crystals were analysed with a light microscopic image analysis system, scanning electron microscopy (SEM), differential scanning calorimetry (DSC) and powder X-ray diffraction (XRPD) in order to obtain images of the crystal shape, roundness and structure. The residual solvent content of the crystallized products was investigated by a headspace gas chromatographic method.

\section{Materials and methods}

\subsection{Materials}

The following components were used in the experimental work: glycine and ethanol 96\% supplied by VWR Hungary; neutral oil (Miglyol 812) purchased from Sasol Germany GmbH; and purified water (Ph. Eur. quality).

\subsection{Impinging jet crystallization}

Crystallization experiments were carried out in a $250 \mathrm{~mL}$ round-bottomed, double-walled Schmizo crystallization reactor (Schmizo AG, Oftringen, Switzerland) equipped with an IKA Eurostar digital mixer (IKA-Werke GmbH \& Co., Staufen, Germany).

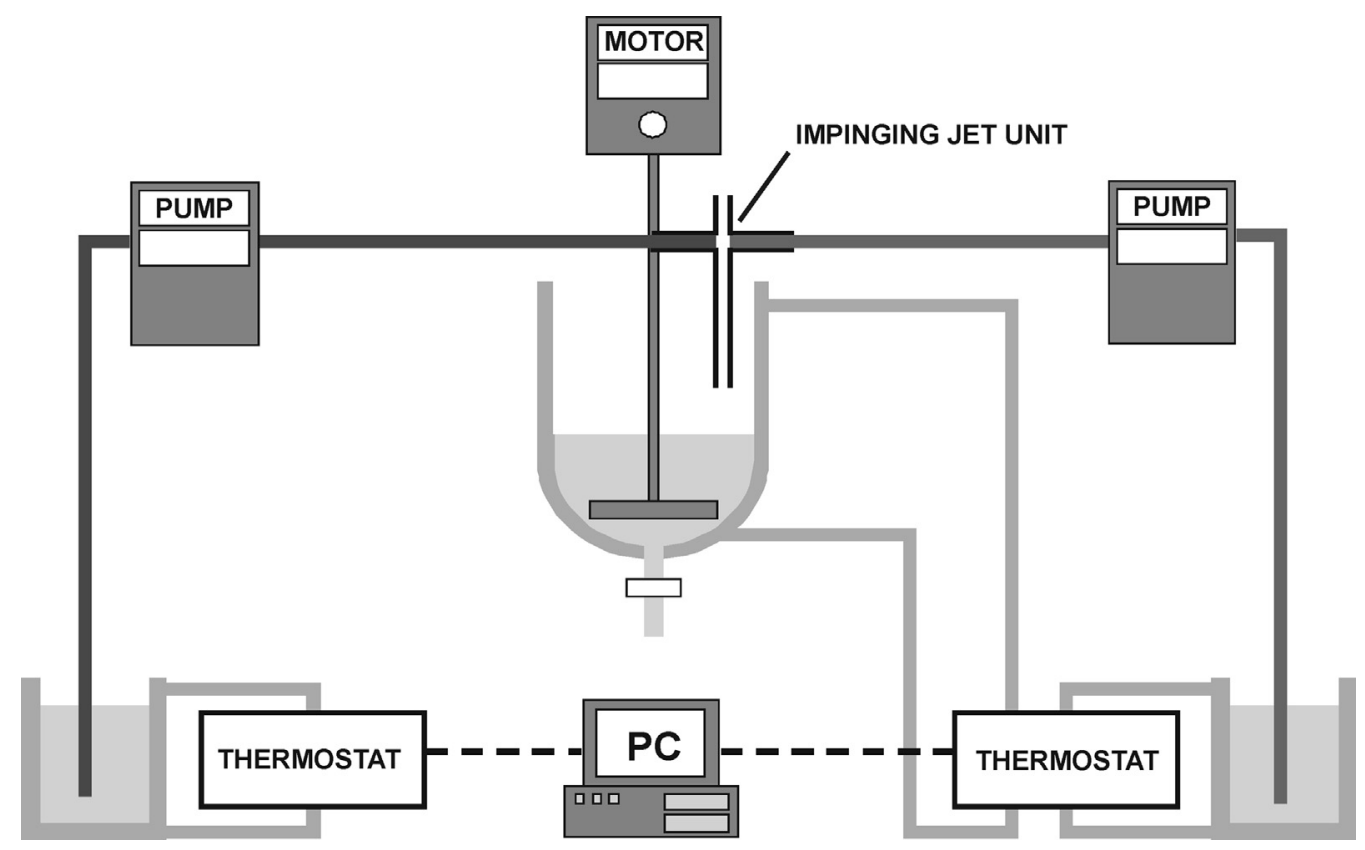

Fig. 1. Experimental apparatus with an impinging jet unit applied in non-submerged mode. 
Temperatures were adjusted with a Thermo Hake P5/C10 (Thermo Haake, Karlsruhe, Germany) thermostat and a Julabo F32 (Julabo GmbH, Seelbach, Germany) cryothermostat controlled by Julabo EasyTemp 2.3e software. Two calibrated Rollpump Type 5198 peristaltic pumps (MTA Kutesz, Budapest, Hungary) were used for liquid feeding. The impinging jet unit was a locally developed device equipped with variable-diameter nozzles, used in non-submerged mode.

During the experiments, the two pumps dosed a nearly saturated aqueous solution of glycine at $25^{\circ} \mathrm{C}(10.02 \mathrm{~g}$ of glycine dissolved in $50 \mathrm{~mL}$ of purified water) and the required amount of ethanol at defined temperatures. The stirring speed was $250 \mathrm{rpm}$. The crystallized products were filtered in porcelain filter and washed with $96 \%$ ethanol. After vacuum drying $\left(40^{\circ} \mathrm{C}, 24 \mathrm{~h}\right)$, the products were stored under normal conditions in closed containers. The experimental apparatus is outlined in Fig. 1.

\subsection{Factorial design}

A $3^{2}$ full factorial design was applied to choose the relevant factors. In series A the influence of the linear velocity and the post mixing time, and in series $B$ the influence of the temperature difference and the post mixing time were investigated on three operational parameters: roundness, $\mathrm{d}(0.5)$ and $\mathrm{D}[4,3]$.

The levels of the factors can be found in Table 1 , while the samples are designated in Table 2. The experiments were performed in randomized sequence. The following approach, containing the interactions of the factors, was used to determine the response surface and the relative effects of the factors $(b)$ :

$y=b_{0}+b_{1} x_{1}+b_{2} x_{2}+b_{3} x_{1}^{2}+b_{4} x_{2}^{2}+b_{5} x_{1} x_{2}$

Statistica for Windows 11 AGA software (StatSoft, Inc. Tulsa, USA) was used for the calculations. During the mathematical evaluations, the confidence interval was $95 \%$, i.e. the differences were significant if $p<0.05$.

\subsection{Differential scanning calorimetry}

The DSC analysis was carried out with a Mettler Toledo STAR thermal analysis system, version 9.30 DSC 821e (Mettler-Toledo AG, Greifensee, Switzerland), at a linear heating rate of $10^{\circ} \mathrm{C} \mathrm{min}^{-1}$, with argon as carrier gas $\left(100 \mathrm{~mL} \mathrm{~min}^{-1}\right)$. The sample weight was in the range $2-5 \mathrm{mg}$ and examinations were performed in the
Table 1

Values of factors.

\begin{tabular}{llll}
\hline Factor & Low level $(-)$ & Mid level $(0)$ & High level $(+)$ \\
\hline Linear velocity $\left(\mathrm{m} \mathrm{s}^{-1}\right)$ & 1.41 & 2.77 & 4.06 \\
Post mixing time $(\mathrm{min})$ & 0 & 5 & 10 \\
Temperature difference $\left({ }^{\circ} \mathrm{C}\right)$ & 0 & 12.5 & 25 \\
\hline
\end{tabular}

temperature interval $25-300^{\circ} \mathrm{C}$, in a sealed $40 \mu \mathrm{L}$ aluminium crucible having three leaks in the lid.

\subsection{X-ray powder diffractometry}

Crystal structures were verified by measuring the X-ray powder diffraction patterns of crystallized samples and the initial material with a Bruker D8 Advance diffractometer (Bruker AXS GmbH, Karlsruhe, Germany) and compared with the structures in the Cambridge Structural Database (Cambridge Crystallographic Data Centre, CCDC, Cambridge, UK). The experiments were performed in symmetrical reflection mode with $\mathrm{Cu}$ K $\alpha$ radiation $(\lambda=1.5406 \AA$ ), using Göbel Mirror bent gradient multilayer optics. The scattered intensities were measured with a Våntec-1 line detector. The angular range was from $3^{\circ}$ to $40^{\circ}$ in steps of $0.007^{\circ}$. Other measurement conditions were as follows: target, $\mathrm{Cu}$; filter, $\mathrm{Ni}$; voltage, $40 \mathrm{kV}$; current, $40 \mathrm{~mA}$; measuring time, $0.1 \mathrm{~s}$ per step.

\subsection{Investigation of crystal shape and roundness}

The crystal shape and roundness of the crystallized products were measured with the LEICA Image Processing and Analysis System (LEICA Q500MC, LEICA Cambridge Ltd., Cambridge, UK). The particles were described in terms of their length, breadth, surface area, perimeter and roundness, which is a shape factor giving a minimum value of unity for a circle. This is calculated from the ratio of the perimeter squared to the area. The adjustment factor of 1.064 corrects the perimeter for the effect of the corners produced by the digitization of the image:

Roundness $=\frac{\text { Perimeter }^{2}}{4 \cdot \pi \cdot \text { Area } \cdot 1.064}$

Table 2

Designation of samples.

\begin{tabular}{|c|c|c|c|}
\hline Sample code & Linear velocity $\left(\mathrm{m} \mathrm{s}^{-1}\right)$ & Post mixing time (min) & Temperature difference $\left({ }^{\circ} \mathrm{C}\right)$ \\
\hline Series A & $\mathrm{x}_{1}$ & $\mathrm{x}_{2}$ & - \\
\hline A1 & 1.41 & 0 & 0 \\
\hline A2 & 1.41 & 5 & 0 \\
\hline A3 & 1.41 & 10 & 0 \\
\hline A4 & 2.77 & 0 & 0 \\
\hline A5 & 2.77 & 5 & 0 \\
\hline A6 & 2.77 & 10 & 0 \\
\hline A7 & 4.06 & 0 & 0 \\
\hline A8 & 4.06 & 5 & 0 \\
\hline A9 & 4.06 & 10 & 0 \\
\hline Series B & - & $\mathrm{x}_{2}$ & $\mathrm{x}_{1}$ \\
\hline B1 & 2.77 & 0 & 0 \\
\hline B2 & 2.77 & 5 & 0 \\
\hline B3 & 2.77 & 10 & 0 \\
\hline B4 & 2.77 & 0 & 12.5 \\
\hline B5 & 2.77 & 5 & 12.5 \\
\hline B6 & 2.77 & 10 & 12.5 \\
\hline B7 & 2.77 & 0 & 25 \\
\hline B8 & 2.77 & 5 & 25 \\
\hline B9 & 2.77 & 10 & 25 \\
\hline
\end{tabular}


Table 3

Crystallization results in series A.

\begin{tabular}{|c|c|c|c|c|c|c|}
\hline Sample & Linear velocity $\left(\mathrm{m} \mathrm{s}^{-1}\right)$ & Post mixing time (min) & Roundness & $\mathrm{d}(0.5)(\mu \mathrm{m})$ & $\mathrm{D}[4,3](\mu \mathrm{m})$ & Percentage yield \\
\hline $\mathrm{A} 1$ & 1.41 & 0 & 2.977 & 15.792 & 21.497 & 62.84 \\
\hline A2 & 1.41 & 5 & 2.292 & 15.808 & 20.295 & 65.90 \\
\hline A3 & 1.41 & 10 & 2.089 & 31.222 & 37.498 & 67.22 \\
\hline A4 & 2.77 & 0 & 2.715 & 16.770 & 22.530 & 65.17 \\
\hline A5 & 2.77 & 5 & 2.251 & 26.057 & 31.610 & 64.14 \\
\hline A6 & 2.77 & 10 & 2.033 & 34.285 & 40.650 & 66.10 \\
\hline A7 & 4.06 & 0 & 2.116 & 14.029 & 17.175 & 64.97 \\
\hline A8 & 4.06 & 5 & 2.363 & 13.778 & 17.784 & 68.06 \\
\hline A9 & 4.06 & 10 & 1.961 & 31.948 & 38.076 & 68.72 \\
\hline
\end{tabular}

Table 4

Crystallization results in series B.

\begin{tabular}{|c|c|c|c|c|c|c|}
\hline Sample & Temperature difference $\left({ }^{\circ} \mathrm{C}\right)$ & Post mixing time (min) & Roundness & $\mathrm{d}(0.5)(\mu \mathrm{m})$ & $\mathrm{D}[4,3](\mu \mathrm{m})$ & Percentage yield \\
\hline B1 & 0 & 0 & 1.825 & 10.142 & 13.329 & 82.25 \\
\hline B2 & 0 & 5 & 2.429 & 9.249 & 11.241 & 81.11 \\
\hline B3 & 0 & 10 & 2.935 & 9.368 & 11.563 & 80.54 \\
\hline B4 & 12.5 & 0 & 2.851 & 8.335 & 10.889 & 81.54 \\
\hline B5 & 12.5 & 5 & 2.186 & 8.524 & 10.803 & 85.59 \\
\hline B6 & 12.5 & 10 & 2.292 & 9.664 & 12.662 & 82.34 \\
\hline B7 & 25 & 0 & 2.071 & 8.575 & 11.599 & 80.60 \\
\hline B8 & 25 & 5 & 2.166 & 10.204 & 13.849 & 85.73 \\
\hline B9 & 25 & 10 & 2.513 & 8.835 & 11.636 & 84.33 \\
\hline
\end{tabular}

The products were suspended in Miglyol 812 with ultrasound in order to ensure the presence of individual particles. Approximately 1000 particles per sample were examined.

\subsection{Scanning electron microscopy}

The morphology of the particles was examined by SEM (Hitachi S4700, Hitachi Scientific Ltd., Tokyo, Japan). A sputter coating apparatus (Bio-Rad SC 502, VG Microtech, Uckfield, UK) was applied to induce electric conductivity on the surface of the samples. The air pressure was 1.3-13.0 $\mathrm{mPa}$.

\subsection{Particle size distribution analysis}

A Malvern Mastersizer laser diffraction analyser (Malvern Instruments Ltd., Malvern, UK) with a measuring range of 0.02$2000 \mu \mathrm{m}$ was used to measure the crystal size distributions. The particle size distribution was determined in a dry method with a Scirocco dry powder feeder; air was used as the dispersion agent.

At least three repeated measurements were performed on each sample and the mean value was calculated. The tables with the results contain $d(0.5)$ (defined as the diameter where half of the population lies below this value) and $\mathrm{D}[4,3]$ (the mean diameter over volume, also referred to as the DeBroukere mean).

\subsection{Determination of residual solvent content}

The residual solvent content was analysed by a headspace gas chromatographic method, with an Agilent 7890 A gas chromatograph (Agilent Technologies Inc., Santa Clara, CA, USA) with a DB624 capillary column $(60 \mathrm{~m} \times 0.25 \mathrm{~mm} \times 1.4 \mu \mathrm{m}$, nominal $)$ equipped with an Agilent GC Sampler 80 and a flame ionization detector. The conditions of the gas chromatographic analysis were as follows: the oven temperature was initially maintained at $40^{\circ} \mathrm{C}$ for $6 \mathrm{~min}$, and then raised at a rate of $7^{\circ} \mathrm{C} \mathrm{min}{ }^{-1}$ to $194^{\circ} \mathrm{C}$, where it was held for $0 \mathrm{~min}$. The temperature of the injector was set at $220^{\circ} \mathrm{C}$ and the detector temperature was set at $300^{\circ} \mathrm{C}$. Helium was used as carrier gas at a pressure of $34.8 \mathrm{psi} .1 \mathrm{~mL}$ samples were injected in split mode, with a split ratio of $8: 1$. The agitator temperature was $80^{\circ} \mathrm{C}$ with a speed of $500 \mathrm{rpm}$, and the syringe temperature was $110^{\circ} \mathrm{C}$. The sample equilibration time was $20 \mathrm{~min}$. The hydrogen gas and air flow rates were 30 and $350 \mathrm{~mL} \mathrm{~min}^{-1}$, respectively.

The ethanol concentration of the standard solution was $100 \mu \mathrm{g} \mathrm{mL}^{-1}$. The blank sample contained $500 \mathrm{mg}$ of sodium sulfate dissolved in $1 \mathrm{~mL}$ of water for injection in a $20 \mathrm{~mL}$ headspace vial. The standard sample contained the same quantity of sodium sulfate dissolved in $1 \mathrm{~mL}$ of standard solution, while the sample solution contained $500 \mathrm{mg}$ of sodium sulfate and $100 \mathrm{mg}$ of crystallized glycine sample dissolved in $1 \mathrm{~mL}$ of water for injection.
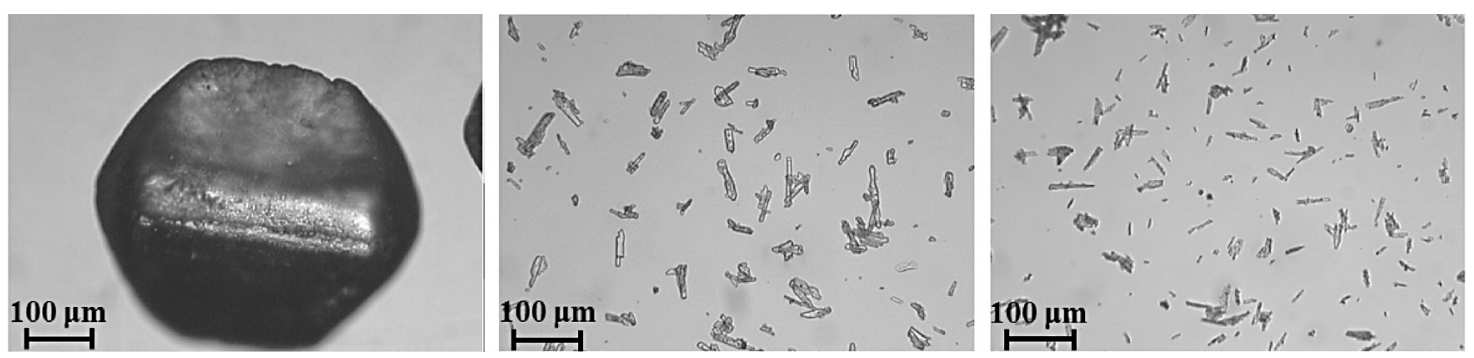

Fig. 2. Light microscopy images of glycine crystals.

(left: original crystal; middle: crystallized product A8; right: crystallized product B7). 

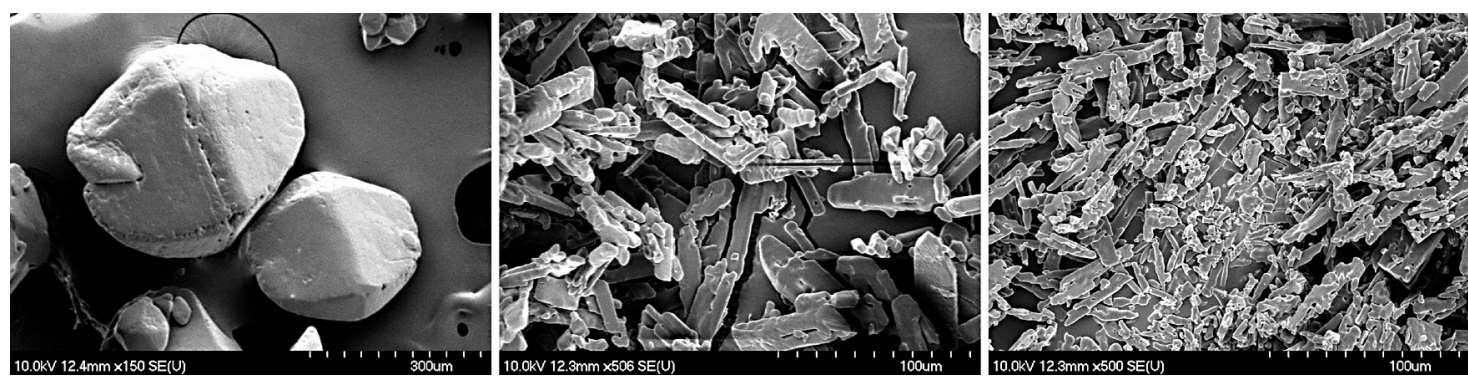

Fig. 3. SEM images of glycine crystals.

(left: original crystal; middle: crystallized product A8; right: crystallized product B7).

\section{Results and discussion}

The crystallization results for the two series are presented in Tables 3 and 4. Each assay was repeated three times; the Tables show the average results of roundness, $\mathrm{d}(0.5), \mathrm{D}[4,3]$ and percentage yield.

The application of the impinging jet crystallization technique resulted in a smaller particle size as compared with the previously investigated conventional crystallization methods. The parallel crystallization processes with the same parameters produced the same particle size distribution, which confirmed the reproducibility of the method. In series A, increase of the post-mixing time improved the roundness, but increased the particle size of the

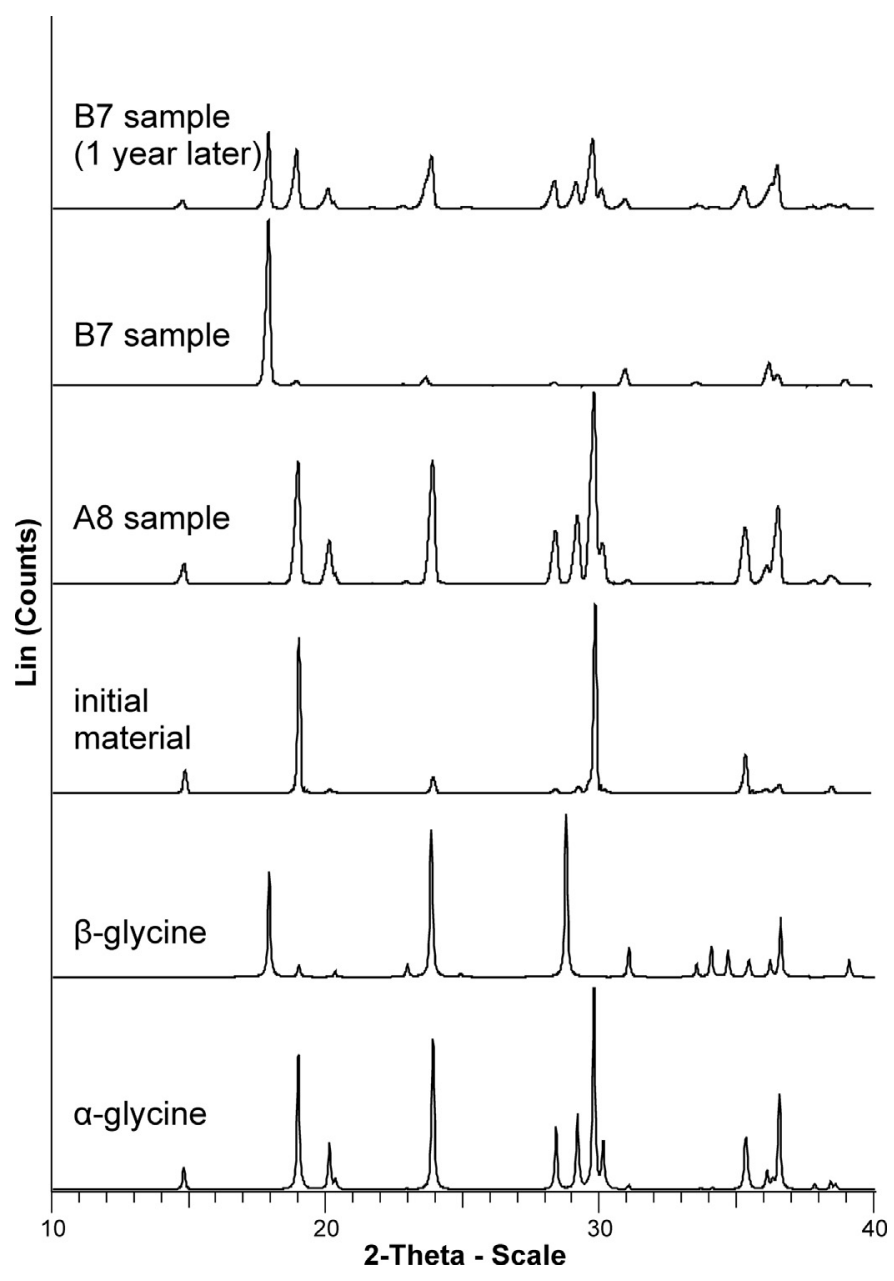

product, which was in contrast with the announced goal. The average particle size increased to a greater extent particularly at a post-mixing time of $10 \mathrm{~min}$. As the crystallization parameters had opposite effects on the particle size and roundness, it was favourable to apply a post-mixing time reduction. In series $\mathrm{B}$, neither the temperature difference nor the post-mixing time influenced the particle size or roundness of the crystallized products significantly, but each individual parameter setting resulted in significantly smaller particles as compared with series A. The percentage yield in series B was higher due to the lower

Exo up

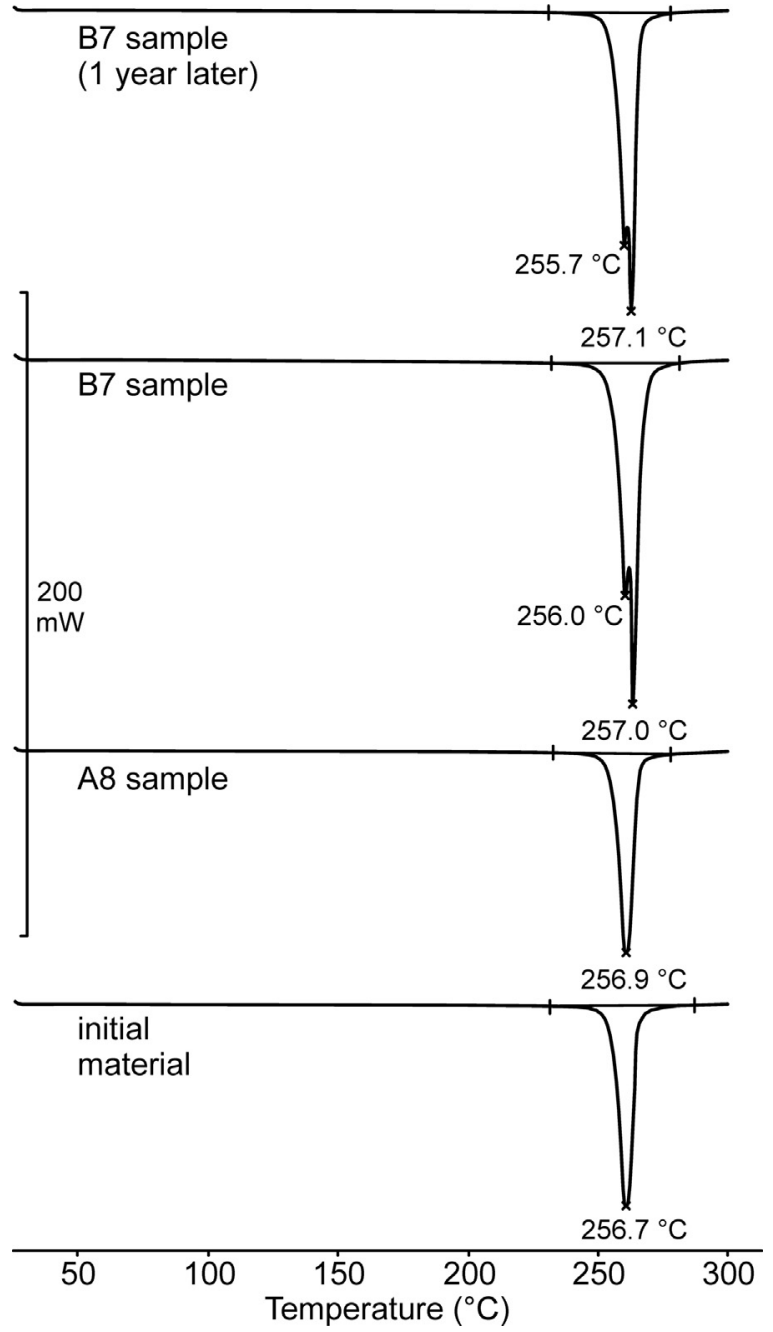

Fig. 5. DSC thermograms of the initial material and crystallized products. 
Table 5

Factorial design results (series A).

\begin{tabular}{lll}
\hline Dependent variable & Polynomial function & $\mathrm{r}^{2}$ \\
\hline Roundness & $y=2.32-0.31 \mathrm{x}_{1}-\underline{0.58 \mathrm{x}_{2}}+0.04 \mathrm{x}_{1}^{2}-0.01 \mathrm{x}_{2}^{2}+0.37 \mathrm{x}_{1} \mathrm{x}_{2}$ & 0.858 \\
$\mathrm{~d}(0.5)$ & $y=22.19-1.02 \mathrm{x}_{1}+\underline{16.94 \mathrm{x}_{2}}+5.29 \mathrm{x}_{1}^{2}-5.46 \mathrm{x}_{2}^{2}+1.25 \mathrm{x}_{1} \mathrm{x}_{2}$ & 0.937 \\
$\mathrm{D}[4,3]$ & $y=27.47-2.09 \mathrm{x}_{1}+\underline{18.32 \mathrm{x}_{2}}+6.24 \mathrm{x}_{1}^{2}-6.34 \mathrm{x}_{2}^{2}+2.45 \mathrm{x}_{1} \mathrm{x}_{2}$ & 0.943 \\
\hline
\end{tabular}

solubility of glycine in the $1: 2$ solvent-antisolvent mixture. The filterability of all the crystallized products was satisfactory.

The differences in crystal size and morphological parameters can be seen in the light microscopy and SEM images (Figs. 2 and 3). The original glycine contained large isodimensional crystals with a smooth surface. By contrast, the products with the smallest average particles in the two crystallization series consisted of small, irregular-shaped, needle-form crystals with a smooth surface and poorer roundness. The crystallized products exhibited a slight tendency to aggregate due to the small particle size, but this did not cause any problem for the laser diffraction particle size analysis measurements and allowed the application of the dry method.

The polymorphism of the initial material and the products was examined with a powder X-ray diffraction apparatus and compared with the structures in the Cambridge Structural Database (refcodes GLYCIN02 $(\alpha)$ and $\operatorname{GLYCIN}(\beta)$ ) (Fig. 4). It was found that both the initial material and the series A products consisted of the pure stable $\alpha$ polymorph. In contrast, the series B products contained mostly the less stable $\beta$ polymorph, and a small amount of the $\alpha$ polymorph. According to the literature data (Weissbuch et al., 2005; Ferrari et al., 2003), the appearance of the $\beta$ polymorph is caused by the presence (and the concentration) of ethanol in the crystallization process. While the $1: 1$ solventantisolvent ratio favoured the formation of the stable $\alpha$ form, the higher ethanol ratio resulted in the appearance of the less stable $\beta$ polymorph.

Transformation of the $\beta$ form into the $\alpha$ polymorph began during storage. The pure $\beta$ form had been generated as described in the literature (Boldyreva et al., 2003a). Powder mixtures of various compositions $(0+100,10+90,20+80 \alpha+\beta$ forms, and so on) were prepared from the two polymorphs and the calibration curve was recorded. The calibration curve based on the

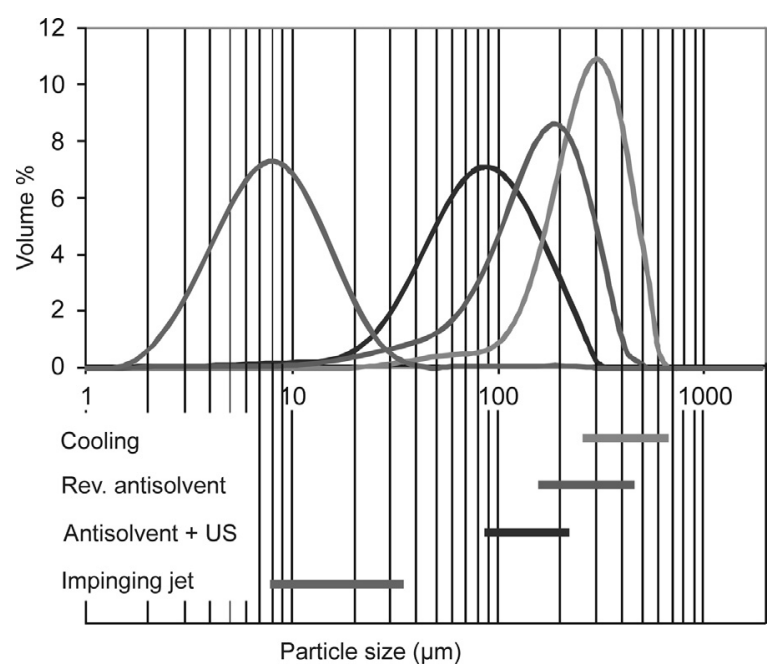

Fig. 6. Particle size distribution and average particle size range produced by different crystallization methods.

(top: particle size distribution of the product with the smallest average particle size achieved with the given method; bottom: average particle size ranges $(\mathrm{d}(0.5))$ attained with the given method). characteristic peak area of the $\alpha$ form (peaks at 29.225, 29.827 and $30.1722 \theta$ ) was as follows: $\mathrm{y}(\alpha$ form $\%)=-1.7465 \mathrm{x}$ (net area $)+158.25\left(R^{2}=0.991\right)$. The initial $\beta$ form content of the series B samples was between 72 and $96 \%$. After 1 year of storage, the $\beta$ form content had decreased to $13-17 \%$. The series A products did not change during this storage period. It was found that the $1: 1$ solvent ratio used in the crystallization processes was crucial for the formation of the stable polymorphic form.

DSC studies confirmed the results of the powder X-ray analysis. The thermograms of the initial material and the series A products contained one endothermic peak at about $257^{\circ} \mathrm{C}$, which corresponds to the melting point of the $\alpha$ form (Srinivasan, 2008). In contrast, the thermograms of the series B samples displayed two endothermic peaks. The lower-temperature peak corresponded to the melting point of the $\beta$ form, while the second peak was caused by the melting of the $\alpha$ form. It was not possible to specify the proportion of the polymorphs because the two endothermic peaks overlapped. After storage for one year, the thermograms of the series B samples were similar to the previously recorded ones. It has been reported that the phase transition of the $\gamma$ form to the $\alpha$ polymorph causes a small endothermic peak at about $179{ }^{\circ} \mathrm{C}$ (Srinivasan, 2008). Our results indicated that our samples did not contain any $\gamma$ form (Fig. 5).

The growth of the crystals during impinging jet crystallization is rapid, due to the homogeneous and high degree of supersaturation, so that the chance of the occurrence of solvent inclusion is high. Ethanol (used as antisolvent) belongs in the ICH $\mathrm{Q} 3 \mathrm{C}(R 2)$ Guideline Class 3 group (where the residual solvent concentration is at most $5000 \mathrm{ppm}$ ), and it was therefore necessary to determine its concentration in the crystallized products (ICH, 2011). The residual solvent contents of the crystallized samples were determined by headspace gas chromatography (Grodowska and Parczewski, 2010). Our results indicated that the ethanol content of the initial sample was less than the limit of quantification, and it was therefore assumed that ethanol was not used in the preparation of this material. The maximum residual solvent content of the series A samples was $9 \mathrm{ppm}$, while the samples in series B contained a maximum $145 \mathrm{ppm}$ of ethanol. The measured residual solvent content of the samples was low relative to the maximum values prescribed in the $\mathrm{ICH}$ requirements, which demonstrated the applicability of the impinging jet method in the antisolvent crystallization of glycine despite the extremely rapid nucleation.

Statistical analysis results relating to the effects of the crystallization parameters on the roundness and particle size are presented in Table 5, where the statistically significant factors are underlined.

In the case of series A, only the post-mixing time exhibited a significant linear relationship with the changes in roundness, $d$ $(0.5)$ and $D[4,3]$ results (the response surface $r^{2}$ results were 0.858 , 0.937 and 0.943 , respectively). Neither the linear nor the quadratic relationship of the linear velocity and the interaction effect of the two independent variables displayed a significant effect on the change in these dependent variables. An increase of the postmixing time increased the average particle size, but reduced the roundness, and the post-mixing time therefore had to be reduced to achieve the desired small particles. We assume that an increase 
of the linear velocity would cause a further particle size reduction, but the pump capacity was limited, so that the velocity could not be increased as compared with the original parameters described in the crystallization studies. The investigated parameters did not cause significant changes in the particle size and roundness in series $\mathrm{B}$.

Fig. 6 depicts the particle size distributions of the products with the smallest particles and the average particle size ranges produced by impinging jet crystallization and the previously investigated crystallization methods (Aigner et al., 2012). Those studies had shown that the largest particles were achieved by conventional cooling crystallization. The reverse antisolvent and antisolvent methods with the application of ultrasound were also able to achieve slight reductions in the average particle size of glycine. The impinging jet technology resulted in a further one order of magnitude reduction in particle size.

\section{Conclusions}

Glycine crystals grow rapidly and the crystal size is typically quite large, and glycine is therefore an ideal model material for particle size reduction experiments. Application of the impinging jet method in antisolvent crystallization led to a reproducible decrease in the average particle size of glycine, with suitable low residual organic solvent quantity and roundness. A factorial design was applied to choose the relevant crystallization factors, and the results were analysed by means of a statistical program. The average particle size was an order of magnitude smaller (d $(0.5)=8-35 \mu \mathrm{m})$ as compared with the results of several other crystallization methods (cooling, reverse antisolvent and antisolvent crystallization with the application of ultrasound, where $d$ (0.5) was between 82 and $680 \mu \mathrm{m}$ ). Production of the stable polymorphic form required the application of a 1:1 water-ethanol ratio.

The impinging jet crystallization method has proved to be a good tool for optimizing and controlling the nucleation and crystallization of organic materials such as glycine. Furthermore, it can be used as a very effective size reduction method to attain a final product with suitable crystal morphology and a narrow particle size distribution.

\section{Acknowledgements}

The authors wish to thank Rita Ambrus for her contribution to the SEM analysis. We are grateful for the support of DAAD-MÖB project No. 39349.

\section{References}

Aigner, Z., Szegedi, Á., Szabadi, V., Ambrus, R., Sovány, T., Szabó-Révész, P., 2012. Comparative study of crystallization processes in case of glycine crystallization. Acta Pharmaceutica Hungarica 82, 61-68.

Bauer-Brandl, A., 1996a. Polymorphic transitions of cimetidine during manufacture of solid dosage forms. Int. J. Pharm. 140, 195-206.

Bauer-Brandl, A., 1996b. Erratum to polymorphic transitions of cimetidine during manufacture of solid dosage forms. Int. J. Pharm. 145, 253.
Boldyreva, E.V., Drebushchak, V.A., Drebushchak, T.N., Paukov, I.E., Kovalevskaya, Y A., Shutova, E.S., 2003a. Polymorphism of glycine. Thermodynamic aspects. Part I. Relative stability of the polymorphs. J. Therm. Anal. Calorim. 73, 409-418.

Boldyreva, E.V., Drebushchak, V.A. Drebushchak, T.N., Paukov, I.E., Kovalevskaya, Y. A., Shutova, E.S., 2003b. Polymorphism of glycine. Thermodynamic aspects. Part II. Polymorphic transitions. J. Therm. Anal. Calorim. 73, 419-428.

Calvignac, B., Boutin, O., 2009. The impinging jets technology: a contacting device using a SAS process type. Powder Technol. 191, 200-205.

D’Addio, S.M., Prud'homme, R.K., 2011. Controlling drug nanoparticle formation by rapid precipitation. Adv. Drug. Deliv. Rev. 63, 417-426.

Dong, Y., Ng, W.K., Shen, S., Kim, S., Tan, R.B.H., 2011. Controlled antisolvent precipitation of spironolactone nanoparticles by impingement mixing. Int. J. Pharm. 410, 175-179.

Dubbini, A., Censi, R., Martena, V., Hoti, E., Ricciutelli, M., Malaj, L., Martino, P.D. 2014. Influence of $\mathrm{pH}$ and method of crystallization on the solid physical form of indomethacin. Int. J. Pharm. 473, 536-544.

am Ende, D.J., Brenek, S.J., 2004. Strategies to control particle size during crystallization processes. Am. Pharm. Rev. 7, 98-104.

Ferrari, E.S., Davey, R.J., Cross, W.I., Gillon, A.M., Towler, C.S., 2003. Crystallization in polymorphic systems: the solution-mediated transformation of $\beta$ to $\alpha$ glycine. Cryst. Growth Des. 3, 53-60.

Goryainov, S.V., Boldyreva, E.V., Kolesnik, E.N., 2006. Raman observation of a new $(\zeta)$ polymorph of glycine? Chem. Phys. Lett. 419, 496-500.

Grodowska, K., Parczewski, A., 2010. Organic solvents in the pharmaceutical industry. Acta Pol. Pharm. Drug Res. 67, 3-12.

Hacherl, J.M., Paul, E.L., Buettner, H.M., 2003. Investigation of impinging-jet crystallization with a calcium oxalate model system. AIChE J. 49, 2352-2362.

ICH International Conference on Harmonisation of Technical Requirements for Registration of Pharmaceuticals for Human Use, ICH harmonised tripartite guideline validation of Impurities: Guideline for residual solvents Q3C(R5), (February 2011)

Lin, C.H., Gabas, N., Canselier, J.P., Pèpe, G., 1998. Prediction of the growth morphology of aminoacid crystals in solution: I. è-glycine. J. Cryst. Growth 191, 791-802.

Liu, L.X., Marziano, I., Bentham, A.C., Litster, J.D., White, E.T., Howes, T. 2013. Influence of particle size on the direct compression of ibuprofen and its binary mixtures. Powder Technol. 240, 66-73.

Liu, Y., Cheng, C., Liu, Y., Prud'homme, R.K., Fox, R.O., 2008. Mixing in a multi-inlet vortex mixer (MIVM) for flash nano-precipitation. Chem. Eng. Sci. 63, 28292842.

Louhi-Kultanen, M., Karjalainen, M., Rantanen, J., Huhtanen, M., Kallas, J., 2006. Crystallization of glycine with ultrasound. Int. J. Pharm. 320, 23-29.

Lung-Somarriba, B.L.M., Moscosa-Santillan, M., Porte, C., Delacroix, A., 2004. Effect of seeded surface area on crystal size distribution in glycine batch cooling crystallization: a seeding methodology. J. Cryst. Growth 270, 624-632.

McCausland, L.J., Cains, P.W., 2003. Ultrasound to make crystals. Chem. Indust. 5, 15 16.

McCausland, L.J., Cains, P.W., Martin, P.D., 2001. Use the power of sonocrystallization for improved properties. Chem. Eng. Prog. 97, 56-61.

Midler, M., Paul, E.L., Whittington, E.F., Futran, M., Liu, P.D., Hsu, J., Pan, S.H., 1994 Crystallization method to improve crystal structure and size. Patent US $5,314,506$.

Rabesiaka, M., Sghaier, M., Fraisse, B., Porte, C., Havet, J.-L., Dichi, E., 2010. Preparation of glycine polymorphs crystallized in water and physicochemical characterizations. J. Cryst. Growth 312, 1860-1865.

Srinivasan, K., 2008. Crystal growth of $\alpha$ and $\gamma$ glycine polymorphs and their polymorphic phase transformations. J. Cryst. Growth 311, 156-162.

Srinivasan, K., Devi, K.R., Azhagan, S.A., 2011. Characterization of $\alpha$ and $\gamma$ polymorphs of glycine crystallized from water-ammonia solution. Cryst. Res. Technol. 46, 159-165.

Tung, H.-H., Paul, E.L., Midler, M., McCauley, J.A., 2009. Crystallization of Organic Compounds - An Industrial Perspective. John Wiley \& Sons Inc., New Jersey, pp. 196-204.

Weissbuch, I., Torbeev, V.Y., Leiserowitz, L., Lahav, M., 2005. Solvent effect on crystal polymorphism: why addition of methanol or ethanol to aqueous solutions induces the precipitation of the least stable $\beta$ form of glycine. Angew. Chem. Int. Ed. 117, 3290-3293.

Woo, X.Y., Tan, R.B.H., Braatz, R.D., 2009. Modeling and computational fluid dynamics - population balance equation - micromixing simulation of impinging jet crystallizers. Cryst. Growth Des. 9, 156-164.

Woo, W.Y., Tan, R.B.H., Braatz, R.D., 2011. Precise tailoring of the crystal size distribution by controlled growth and continuous seeding from impinging jet crystallizers. Cryst. Eng. Comm. 13, 2006-2014. 